\title{
Hybrix: Experimental characterization of a micro-sandwich sheet
}

\author{
A.M. Pimentel ${ }^{\mathrm{a}, \mathrm{b}, *}$, J.L. Alves $^{\mathrm{b}}$, N.M. Merendeiro $^{\mathrm{a}, 1}$, D. Oliveira $^{\mathrm{a}, 2}$ \\ a Centro Tecnológico Sodecia, Rua Eng. Frederico Ulrich, 2650, 4470-605 Maia, Portugal \\ b CMEMS, Universidade do Minho, Campus de Azurém 4800-058 Guimarães, Portugal
}

\section{A R T I C L E I N F O}

\section{Article history:}

Received 18 November 2015

Received in revised form 17 February 2016

Accepted 5 March 2016

Available online 7 March 2016

\section{Keywords:}

Micro-sandwich sheets

Lamera

Hybrix

Uniaxial tensile test

Nakazima test

Forming limit curve

\begin{abstract}
A B S T R A C T
The development of new micro-sandwich sheets (i.e. two metallic skins separated by a composite core), which can be shaped by conventional sheet metal forming processes, became one of the most interesting materials and promising automotive applications in the past few years. However, due to the lack of understanding of certain fundamentals related with the mechanical behavior of micro-sandwich sheets during forming processes, the transfer and scale-up of this promising material to industry has been hindered. To overcome problems concerning the formability of these new materials and make the conventional sheet forming process more adapted, the experimental characterization of the micro-sandwich sheets is absolutely necessary. In this work, a new approach to experimentally characterize micro-sandwich sheets, with the mechanical properties of the core unknown, is presented. Firstly, for the characterization of stress-strain curves and anisotropy, uniaxial tensile tests in 3 different orientations with respect to rolling direction are performed on total micro-sandwich specimens and skin-only specimens. Secondly, the mechanical properties of the core are then deduced from micro-sandwich and skins' mechanical properties, and the constitutive parameters established. Additionally, 5 different Nakazima geometries were punched according to ISO 12004 for formability assessment. Experimental Forming Limit Curves (FLC), punch forces and principal strain data were recorded during the tests using high resolution cameras and system GOM ARAMIS. Finally, the experimental mechanical tests were numerically reproduced. The systematic excellent agreement between numerical and experimental results is a good validation of the methodology proposed in the present work to identify the constitutive properties of the micro-sandwich materials.
\end{abstract}

(C) 2016 Published by Elsevier B.V

\section{Introduction}

Lighter vehicles, lower fuel consumption and easier recyclability is the new pragma of automotive industry due to the most recent environmental concerns. Such new framework has been the opportunity for the introduction of new lighter and challenging materials, such as alternative metals and composites, which have been at the heart of the research and innovation in order to develop and introduce in the market the lighter and more environmentally friendly future vehicles, as stated by Ghassemieh (2011).

In this context, multi-layer materials, usually known as sandwich materials, are currently one of the most promising tech-

\footnotetext{
* Corresponding author at: CMEMS, Universidade do Minho, Campus de Azurém, 4800-058 Guimarães, Portugal.

E-mail address: anthony.pimentel@ct.sodecia.com (A.M. Pimentel).

1 Present Adress: Sodecia-participações Sociais Sgps Sa, Rua do Espido, 164-F, Edificio Via Norte, 4470-177 Maia, Portugal.

2 Present Adress: Efacec-Sistemas de Electrónica SA, Rua Eng. Frederico Ulrich, 4470-605 Maia, Portugal.
}

nological solutions in terms of new advanced materials to be applied in automotive industry. As Moreira et al. (2010) refers, the main advantages of these materials are the excellent stiffness to weight and strength to weight ratios, ease of production and, when developed for this purpose, excellent ability to absorb vibrations and noise.

In the past few years, the sandwich concept has been applied to very thin metallic sheet layers, which can be transformed by conventional sheet forming processes. These sheet materials, also known as micro-sandwich, are usually made by a polymeric soft core (as epoxy resins or rubber) covered by two metallic skins. In certain cases, the soft core can also contain fibbers, metallic or not. Some of these materials have already been used with success in the automotive industry. For instance, the CORUS micro-sandwich Hylite was applied in automotive parts as bonnets, roof panels and hoods. Mann (1999) mentions the NedCar Acess (1996), Palkowski and Lange (2005) refers the Ford e-Ka (2000), and the Hylite supplier Alcan Singen $\mathrm{GmbH}$ claims the successful applications on the Aixam 400 (1997) and Audi A2 (2000). Volkswagen is using the LITECORE micro-sandwich, developed and produced in a part- 
nership with ThyssenKrupp Steel Europe, to the production of a super lightweight hood for the Polo R WRC, as revealed online by TSE (2014). Volvo was also involved in the development of a new micro-sandwich, called Hybrix, born out of a partnership between Lamera $\mathrm{AB}$, a spin-off company from the Sweden OEM, and the Chalmers University. The Hybrix core consists of millions of microscopic stainless steel fibers vertically oriented against the AISI 304L stainless steel skins and bounded by an epoxy resin, as depicted in Fig. 1.

The Hybrix sandwich material can display several thickness combinations, with different nominal thicknesses and symmetric or asymmetric metallic skins, i.e. the two outside metallic skins can exhibit the same or different thicknesses. The experimental results presented in this work concern to a symmetric Hybrix configuration, with the following nominal thicknesses: $1.3 \mathrm{~mm}$ of the core and $0.15 \mathrm{~mm}$ of each metallic skin. Because of its excellent strength and ductility, stainless steel is commonly used in deep drawing parts. So, using stainless steel skins, Hybrix sheets seem to be well adapted to be shaped in sheet forming operations, and very promising to be used in automotive industry.
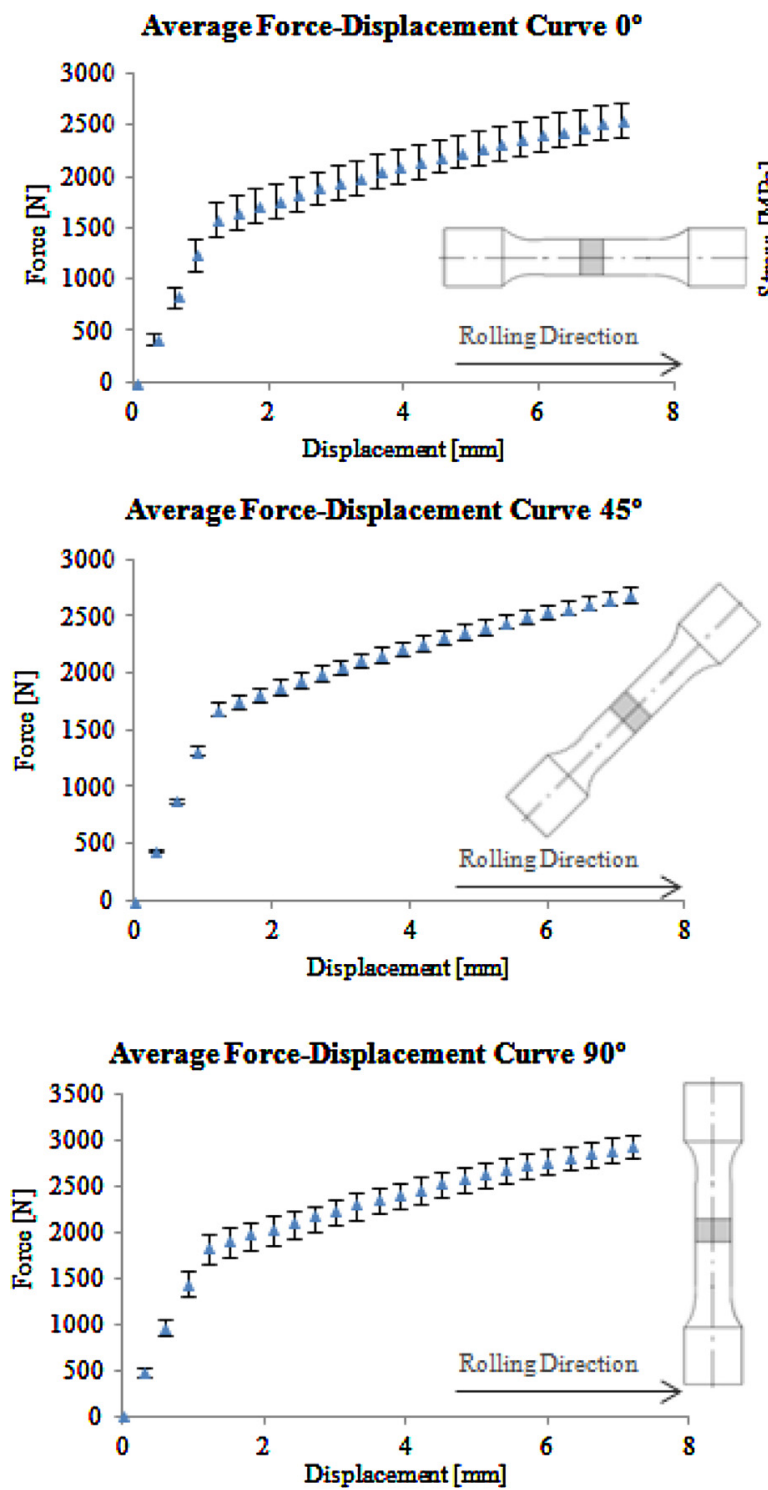

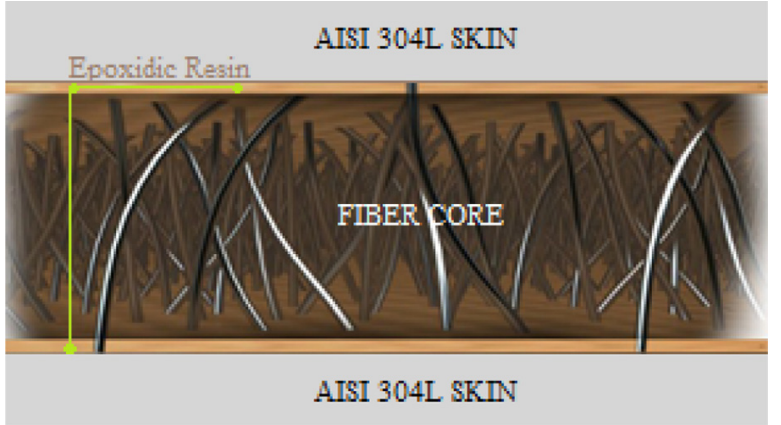

Fig. 1. Scheme of the Hybrix micro-sandwich sheet material, comprising two stainless steel layers and an intermediate composite core (metallic stainless steel fibers embedded in an epoxy resin). The total nominal thickness of $1.6 \mathrm{~mm}$ can be decomposed in two skin layers of $0.15 \mathrm{~mm}$ and a core with $1.3 \mathrm{~mm}$.

To study Hybrix feasibility in automotive industry, several stamped case studies were experimentally tested by Engelmark (2009): the experimental results explored by this author put in evidence a high risk of wrinkles in case of deep drawn parts, which

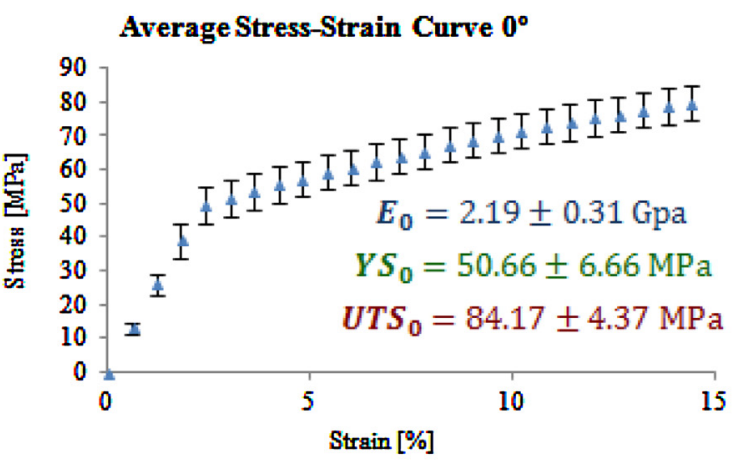

Average Stress-Strain Curve $\mathbf{4 5}^{\circ}$

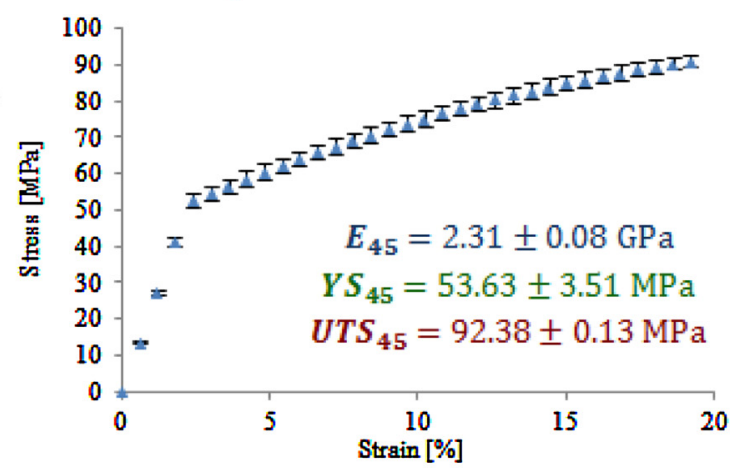

Average Stress-Strain Curve $90^{\circ}$

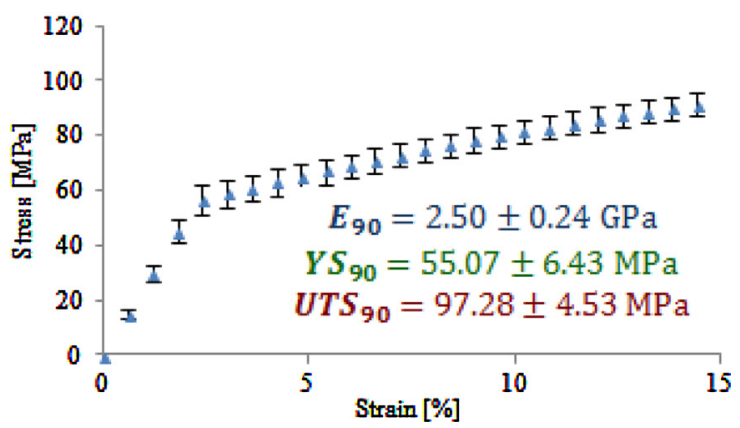

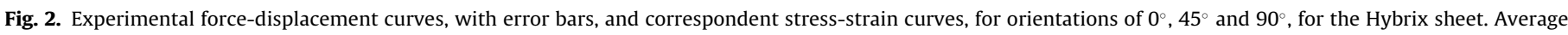
values of Young modulus (E), yield stress (Y) and ultimate tensile strength (UTS) with the respective uncertainty. 

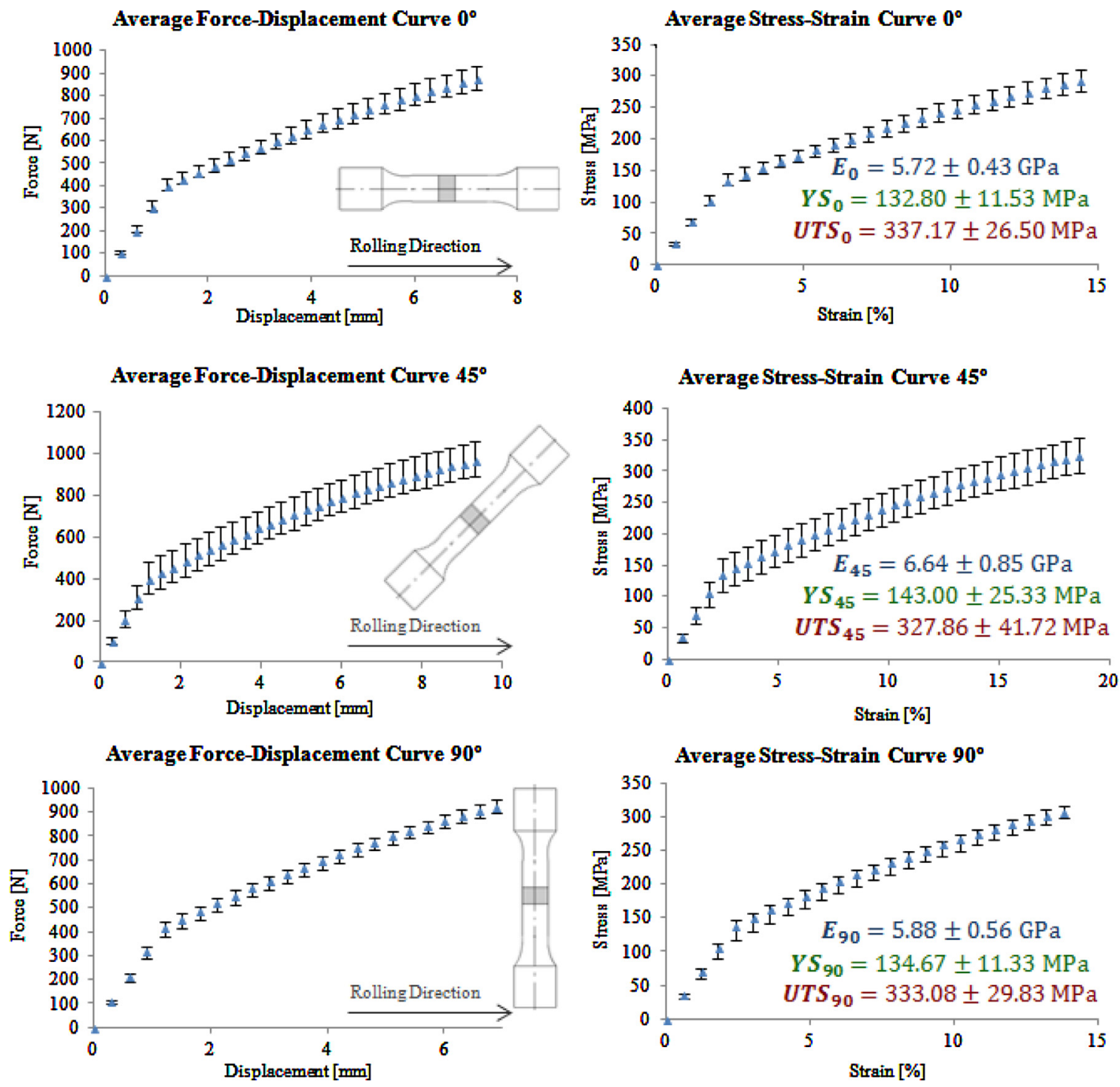

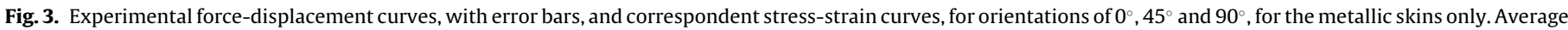
values of Young modulus (E), yield stress (Y) and ultimate tensile strength (UTS) with the respective uncertainty.

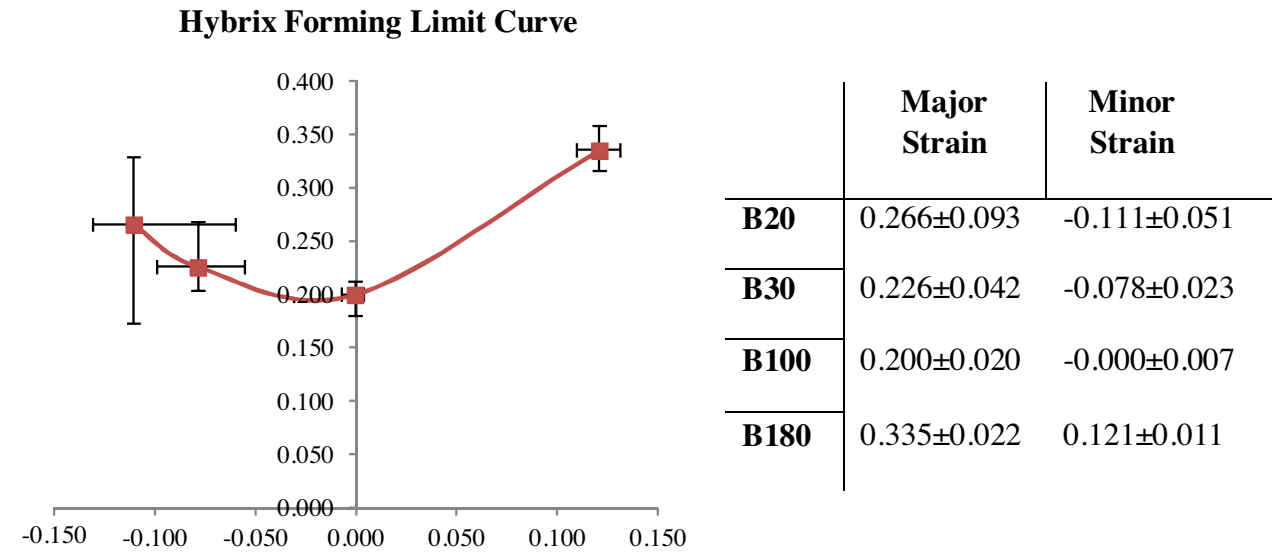

Fig. 4. Experimental Hybrix Forming Limit Curve (FLC), defined with 4 experimental points, with error bars and respective uncertainty.

is a major concern to the stamping process robustness. Moreover, Jackson et al. (2008) conducted some experimental tests in different types of sandwich materials in order to evaluate their formability behavior; in brief, authors claim that, using an incremental forming technique, the Hybrix sandwich material may exhibit some formability problems mainly due to delamination fail- 


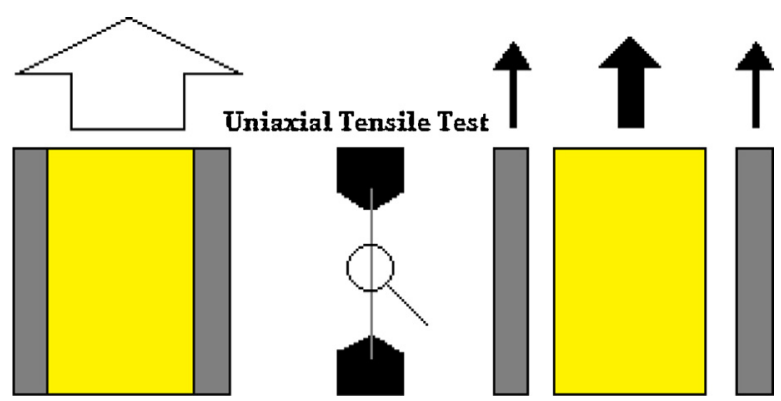

Fig. 5. Decomposition load strategy. The 3 layers (2 metallic skins plus the core) are loaded in parallel during the uniaxial tensile test, and thus the total uniaxial tensile force can be decomposed in two components, the forces supported by the two metallic skins plus the force supported by the core.

ure mode, i.e. separation between the composite polymeric core and the metallic skins. In summary, the previous works in this field have identified several drawbacks concerning the industrialization of micro-sandwich materials. Concerning the conventional cold stamping processes, a major issue is the general poor formability of these materials, and their trend to wrinkling and delamination.

The global mechanical behavior of sandwich materials is obviously related with the thicknesses and mechanical behavior of each layer. Moreover, Palkowski and Lange (2005) compared different sandwich materials and examined their formability, and claim that the difficulties in the deep drawing process of these sandwich system dwells from the different behavior of the layer materials. Nevertheless, it is not quite enough to understand the mechanical behavior of each layer individually given that the global behavior is also driven by the interaction between layers. Moreover, when very thin skins are considered, such as the ones on the micro-sandwich materials, the mechanical properties can change and new constitutive model parameters are needed to accurately describe the formability and springback phenomena, as claimed by Starman et al. (2014). Luzin et al. (2005) compare several experimental techniques to measure the Young Modulus of samples made from sub-millimeter thickness layers, and observed that the elastic properties can no longer be measured with conventional mechanical tests. The manufacture process of multi-layer sheets, even if well controlled, always introduces some variability of material properties and bounding conditions that affects the quality of the stamped parts. Van Den Boscha et al. (2009) also study the relationship between the delamination in a polymer coated metal and the deep-drawing process parameters; the authors consider that both manufacture and stamping processes contributes to delamination and debonding of multi-layer materials affecting its formability.

\section{Core Force-Displacement Curve}

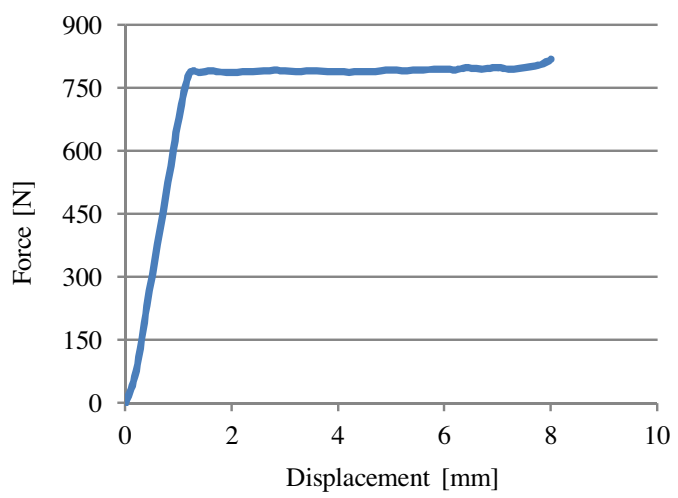

Thus, to adapt the conventional sheet forming processes to the features and mechanical behavior of Hybrix sheets, it is absolutely paramount to carry out their experimental characterization beforehand. Such information is crucial to build robust numerical models to design the forming processes in order to provide reliable and accurate results.

However, the experimental characterization of the Hybrix materials is not a simple task. For instance, the chosen process to cut the testing specimens affects the experimental results. In case of laser cutting, one must consider the fusion and burning of the microsandwich polymeric cores, the heat affected zone in the metallic skins and the presence of burrs along the contour. In case of water jet cutting, the core adhesive properties can be seriously affected by the water and abrasive particles, particularly the epoxy cores. In order to avoid such drawbacks and obtain a better cutting quality surface, Pauchard (2009) suggests the Laser MicroJet technique. Moreover, on the one hand, the experimental tests to identify the mechanical properties of a composite core are very specific and still far from the current reality in industry; on the other hand, the constitutive models to model and describe the mechanical behavior of composite materials are complex and not yet completely implemented in the commercial FE codes dedicated to sheet forming simulation.

This work deals with the experimental characterization and numerical modeling of the mechanical behavior of a microsandwich sheets. The main goal is to present and validate a very simple and expedite methodology to characterize the mechanical behavior of an advanced micro-sandwich material. Uniaxial tensile tests were performed on specimens oriented along 3 different orientations with respect to the rolling direction, in order to characterize the isotropic hardening and anisotropy, and the Nakazima tests were performed to characterize the FLC and for preliminary validation test purposes. The numerical model to validate this methodology was created and tested in commercial software PAM-STAMP 2G 2012.2.

\section{Hybrix: experimental characterization}

The aim of this section is to present the results concerning the experimental characterization of the Hybrix sandwich material, in order to determine the constitutive parameters required for the numerical modeling and numerical simulation. The experimental data must be enough to define the hardening curve, the yield criterion and the forming limit curve (FLC). In case of a sandwich system as Hybrix, this information is needed for each layer. The material model is a paramount aspect to take in account in the numerical simulation of the sheet forming processes; since it rules the

\section{Core Stress-Strain Curve}

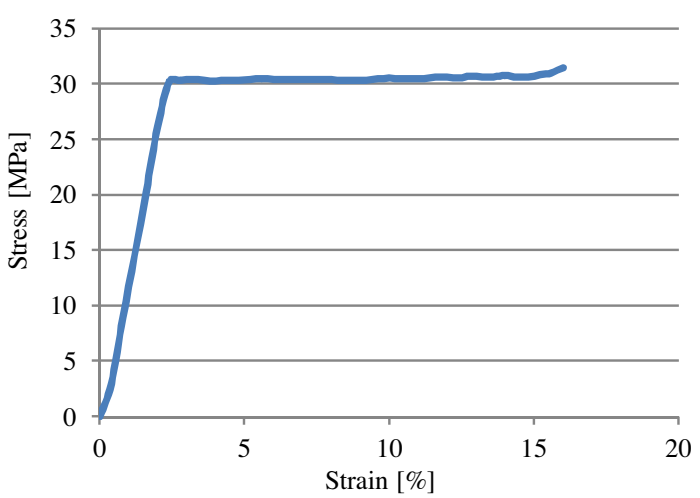

Fig. 6. Deducted core's force-displacement curve and stress-strain curve. 


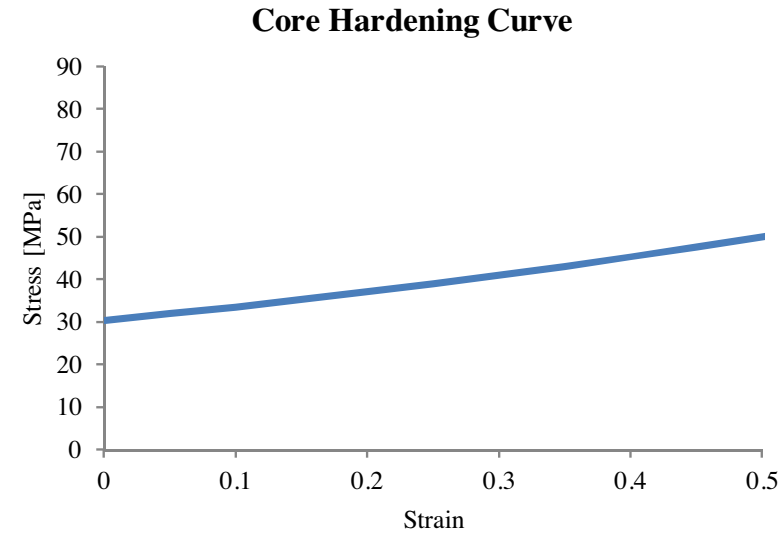

Fig. 7. Resultant core hardening curve identified for the core from the true stressstrain curve.

mechanical behavior of the micro-sandwich materials, the reliability and accuracy of the numerical results depend on it.

\subsection{Uniaxial tensile tests}

Uniaxial tensile tests, commonly used in case of metallic sheets, are able to provide all the relevant experimental data to characterize the hardening and the yield surface. Through the forcedisplacement curves it is possible to establish the stress-strain relationship, and thus to characterize the isotropic hardening curve. Tensile tests carried out for several orientations with respect to the rolling direction are required to characterize the anisotropic behavior and the yield surface. Since Hybrix is a multi-layer material, uniaxial tensile tests can be performed either on the complete micro-sandwich structure or on only the external metallic skins: in what follows both cases, i.e. the complete and skin-only specimens, will be presented and analyzed. Such procedure was adopted in order to isolate and understand the contribution of the fiber/polymeric CORE to the overall mechanical behavior of the Hybrix sandwich material.

The tensile test was conducted according to EN 10002-1: 2009, using a universal testing machine Tinius Olsen and 5 tensile specimens for each orientation with respect to the rolling axis: $0^{\circ}, 45^{\circ}$ and $90^{\circ}$. During the tests, the deformation was measured with a $50 \mathrm{~mm}$ gauge extensometer. All experimental force-displacement curves were recorded. Based on a simple arithmetic average, the force-displacement curves and the correspondent stress-strain curves were averaged for each orientation, and the result are shown in Fig. 2 for the complete Hybrix sheet, and in Fig. 3 for the metallic skins only.
Force-Displacement Curve

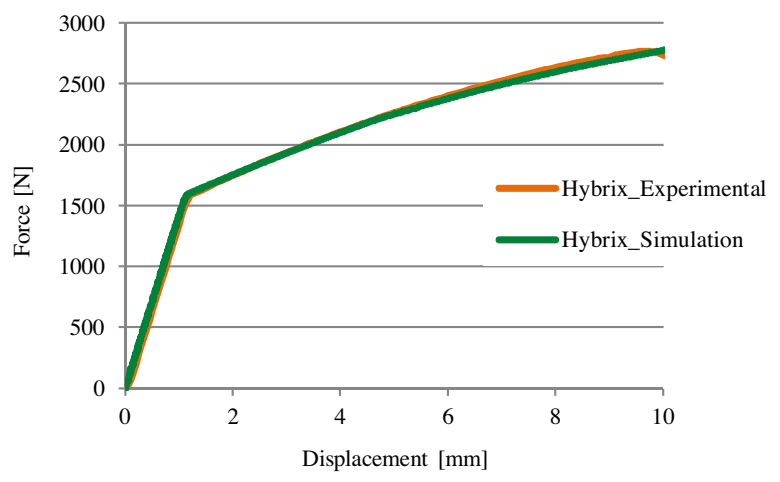

Fig. 9. Comparison between Hybrix experimental force-displacement curve and Hybrix numerical force-displacement curve.

The experimental results of the uniaxial tensile tests with the total Hybrix specimens show that the average yield stress and the average ultimate tensile strength increase with the orientation from $0^{\circ}$ to $90^{\circ}$. The error bars shows that the lowest deviations between the tests were achieved for the orientation of $45^{\circ}$. The elastic behavior and the total elongation between the different orientations are very similar. A final remark concerning the value of the Young modulus, of about $2.3 \mathrm{GPa}$,

The experimental results of the uniaxial tensile tests performed on the metallic skins specimens put in evidence the anisotropic behavior of the metallic skins. In fact, the initial yield stress is higher for $45^{\circ}(143.0 \mathrm{MPa})$, being of $132.8 \mathrm{MPa}$ and $134.7 \mathrm{MPa}$ for $0^{\circ}$ and $90^{\circ}$, respectively. However, it is also true that the highest variation was determined for $45^{\circ}$ specimens, with an uncertainty of about $18 \%$. The elastic strains of the metallic skins and of the total Hybrix specimens are very similar, because the elastic strains are mainly driven by the elastic behavior of the metallic skins. The ductility of the metallic skins tends to be lower than expected for a stainless steel. However, due to the small thickness of the metallic skins it can be expected that the metallic skins are supplied with a pre hardening, with almost all ductility already consumed by the rolling process due to cold reduction. Finally, the experimentally measured Young modulus of around $6 \mathrm{GPa}$ is too low, mainly if compared with the typical value of the Young modulus for AISI 304 stainless steels (193 GPa, matweb.com).

\subsection{Nakazima tests}

Nakazima tests are often used for general formability limits evaluation of metallic materials. With the experimental data of

\section{Hardening Curve 0.15mm Skin}

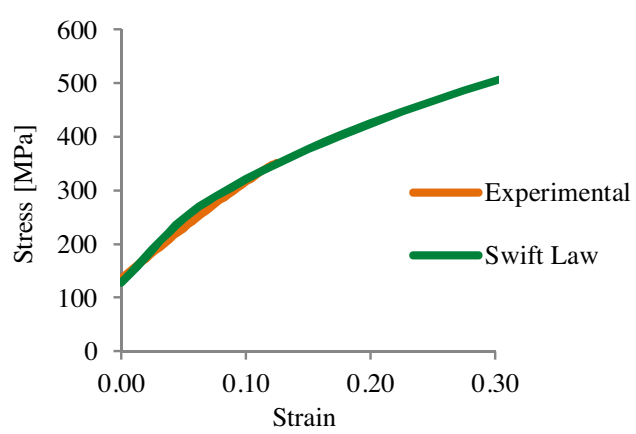

\begin{tabular}{|c|}
\hline Swift Parameters \\
$\mathrm{C}=850 \mathrm{MPa}$ \\
$\mathrm{n}=0.45$ \\
$\varepsilon_{0}=0.015$
\end{tabular}

Fig. 8. Swift parameters for the skin hardening curve definition. Comparison with the experimental stress-strain curve. 
Table 1

Experimental yield forces from: total Hybrix uniaxial tensile test, isotropic core deduction and metallic skin deduction.

\begin{tabular}{|c|c|c|c|c|c|c|c|c|}
\hline & \multicolumn{3}{|c|}{ Yield Force (N) } & \multirow[t]{2}{*}{ Section area $(\mathrm{mm})$} & \multirow[t]{2}{*}{ Gauge length (mm) } & \multicolumn{3}{|c|}{ Elongation $\mathrm{d}(\mathrm{mm})$} \\
\hline & $0^{\circ}$ & $45^{\circ}$ & $90^{\circ}$ & & & $0^{\circ}$ & $45^{\circ}$ & $90^{\circ}$ \\
\hline Total Hybrix & 1583 & 1676 & 1721 & 32 & 50 & 1.2 & 1.2 & 1.2 \\
\hline Skin (1 layer) & 397 & 443 & 466 & 3 & 50 & 1.2 & 1.2 & 1.2 \\
\hline Core & 790 & 790 & 790 & 26 & 50 & 1.2 & 1.2 & 1.2 \\
\hline
\end{tabular}

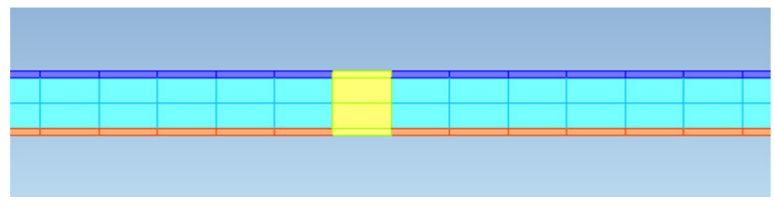

Fig. 10. Finite element mesh with 3D Solid multilayer configuration.

Nakazima test it is possible drawing the Forming Limit Curve (FLC) of the characterized material. The experimental tests were carryed out in an Erichsen (model 142-40) universal testing machine, punching 5 different specimen's geometries (20-30-65-100-180), according to ISO 12004. Each Nakazima specimen refers to a different strain path on the Forming Limit Diagram. To optical 3D deformation measurement purposes, all specimens were painted to display a random speckled pattern. The overall movement of the random speckle pattern was captured during the test by high resolution cameras and treated with the GOM ARAMIS system. The punch velocity was fixed at $20 \mathrm{~mm} / \mathrm{min}$ and the blankholder force fixed at $150 \mathrm{kN}$. The obtained FLC curve is shown in Fig. 4.

Several experimental issues like lens focusing, cameras calibration or the speckle pattern capture, amongst others, can often affect seriously the speckle pattern measurements and mesh generation, and thus invalidate the FLC determination. In the present study, problems with the experimental measurements of the B65 specimens have made impossible its usage. On the other hand, a high variability of the experimentally measured values of both the maximum punch force and principal strains were observed in the narrower specimens, i.e. B20 and B30. Finally, it was found that some specimens started failing unexpectedly too early by the geometry contour nearby the die radius, what is not usually seen in case of monolithic materials. The more stable and robust experimental results were determined for the geometries B100 and B180.

\section{Hybrix: constitutive modelling methodology}

\subsection{Core's stress-strain curves deduction}

To describe numerically the mechanical behavior of a given material it is mandatory to use suitable constitutive models, i.e. flexible enough physically-based laws, and to identify a set of appropriate constitutive parameters. When it concerns to micro-sandwich materials, to know beforehand the constitutive parameters for each material layer, may not be enough or possible. Thus, it is necessary to follow a different strategy which shall allow "determining" the unknown constitutive parameters of the composite material. In case of the Hybrix material, two AISI 304 stainless steel skins are spaced and bonded by an adhesive epoxy resin impregnated with stainless steel micro fibers, which constitutes the core's material. The core's material is a complex composite material, and no experimental data is available for it. The new strategy followed in this work aims at deducting the mechanical properties of the core's material from the previously identified experimental behavior of the total Hybrix material and the single metallic skins, whose results of the uniaxial tensile tests are shown

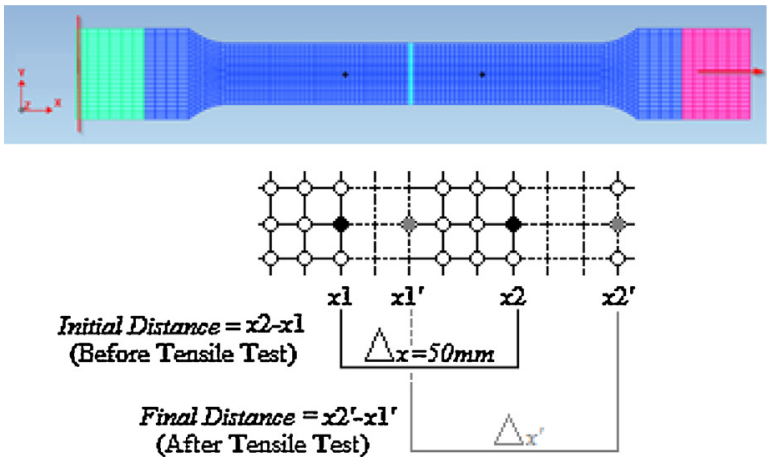

Fig. 11. Control Nodes used to mimic the displacement of the physical extensometer and measure the strain in the specimens after the tensile test.

in Figs. 2 and 3 The assumption behind this strategy is that the total force measured during the uniaxial tensile test of the Hybrix material is the sum of the forces supported by the two skins plus the force supported by the core, as schematically shown in Fig. 5.

The composite fibrous core is hereafter assumed as an isotropic homogeneous material with unknown mechanical properties. Then, it is possible to use the experimental tensile data of both the total Hybrix and the metallic skins to deduct the forcedisplacement and stress-strain curves of the Hybrix core [Fig. 6], considering that

$\vec{F}_{\text {Core }}=\vec{F}_{\text {Hybrix }}-\vec{F}_{\text {Skin }}$

The resultant force-displacement curve and stress-strain curve concerning the core's material are plotted in Fig. 6. It is worth noting the flow stress of about $30 \mathrm{MPa}$ displays almost no hardening, and to compare this value with the one of the metallic skins, of about $132 \mathrm{MPa}$ to $337 \mathrm{MPa}$ (see Fig. 3).

\subsection{Anisotropy and yield criterion}

After determining the stress-strain relationship it is also necessary to define the yield criterion for each layer and for the total Hybrix. Due to the anisotropic behavior of the metallic skins, an anisotropic yield criterion must be used. Therefore, the methodology presented can be followed to deduct and characterize the Hybrix anisotropy, as well to establish a basic analysis to identify the elastic behavior. The experimental force-displacement curves and the global dimensions of the two specimens (metallic skins only and the total Hybrix) are the only data available for the abovementioned deduction.

Table 2

Experimental yield stresses from: total Hybrix uniaxial tensile test, isotropic core deduction and metallic skin deduction.

\begin{tabular}{llll}
\hline Yield stresses & $\sigma 0$ & $\sigma 45$ & $\sigma 90$ \\
\hline Total Hybrix & 51 & 54 & 55 \\
Skin (1 layer) & 135 & 151 & 159 \\
Core & 31 & 31 & 31
\end{tabular}


Table 3

HILL48 constitutive parameters for the total Hybrix, the metallic skins and core.

\begin{tabular}{lllll}
\hline HILL 48 & $H$ & $F$ & $G$ & $N$ \\
\hline Total Hybrix & 1.00 & 0.69 & 1.00 & 3.00 \\
Skin (1 Layer) & 1.00 & 0.45 & 1.00 & 3.00 \\
Core & 1.00 & 1.00 & 1.00 & 3.00 \\
\hline
\end{tabular}

Table 4

Experimental tensile values and adjusted Nakazima values of the Young modulus.

\begin{tabular}{lll}
\hline YOUNG MODULUS & SKIN [GPa] & CORE [GPa] \\
\hline Tensile Test & 6.3 & 1.3 \\
Adjusted to Nakazima test & 22 & 4.5 \\
\hline
\end{tabular}

As previously mentioned, the Hybrix core consists of millions of microscopic stainless steel fibers preferably oriented vertically against the AISI 304L stainless steel skins and bounded by an epoxy resin. Since the stainless steel fibers are assumed vertically oriented in the epoxy matrix, the core's mechanical behavior can be assumed isotropic in the rolling plane, i.e. the same in-plane mechanical behavior irrespective the loading orientation with respect to the rolling direction; in the through-thickness direction, the vertical fibers do not change the compressive behavior. So, the yielding force of the core is considered invariant with the specimens' orientation and equal to $790 \mathrm{~N}$. To maintain the same global behavior, Table 1 displays a new set of skin values for 1 single skin.

Next, it is possible to determine the yield stresses for each orientation from the following expression,

$\sigma_{\theta}=\frac{F_{\theta}}{\mathrm{A}} \times\left(1+\left(\frac{\mathrm{d}}{50}\right)\right)$

The values obtained are depicted in Table 2 .

In general, the anisotropy coefficients are determined from the Lankford coefficients, which are experimentally determined from the width and thickness strains measured during the uniaxial tensile tests. However, due to either the small thickness or the small elongation and ductility of the metallic skins, one were not able of measuring the experimental strain, and thus of determining the Lankford coefficients. Therefore, the anisotropy characterization was carried out taking into account the anisotropy of the yield stresses instead of the anisotropy of the strains. Moreover, the multi-layer character of the total Hybrix material also justifies this option, given that there is no relation between the in-plane strains measured on the metallic skins and the total thickness strain measured on a total Hybrix specimen.

To the description of the surface of plasticity of the metallic skins the HILL48 anisotropic yield criterion was adopted. This yield criterion is expressed by the following quadratic function,

$\bar{\sigma}^{2}=F\left(\sigma_{22}-\sigma_{33}\right)^{2}+G\left(\sigma_{33}-\sigma_{11}\right)^{2}+H\left(\sigma_{11}-\sigma_{22}\right)^{2}+2 L \sigma_{23}^{2}+2 M \sigma_{31}^{2}+2 N \sigma_{12}^{2}$

where $\bar{\sigma}$. is the equivalent tensile stress; $F, G, H, L, M$ and $N$ are constants specifics to the anisotropy of the material, and $\sigma_{i j}, i, j=$ 1,3 . are the stress component of the Cauchy stress tensor. In case of sheet metals, axis 1 is the rolling direction, 2 is the transverse direction and 3 is the normal direction.

As referred by Banabic (2010) it is possible to establish the following mtical relations between the tensile yield stresses and the anisotropy parameters

$\frac{1}{\sigma_{0}^{2}}=G+H ; \frac{1}{\sigma_{45}^{2}}=H+F ; \frac{1}{\sigma_{90}^{2}}=F+G$

and thus to determine the values of the anisotropy parameters $F$, $G$ and $H$. The Table 3 present the HILL48 parameters for the Hybrix micro-sandwich and their two layers, core and skins. Because there is no experimental data to determine parameter $N$, the isotropic case was considered. b180

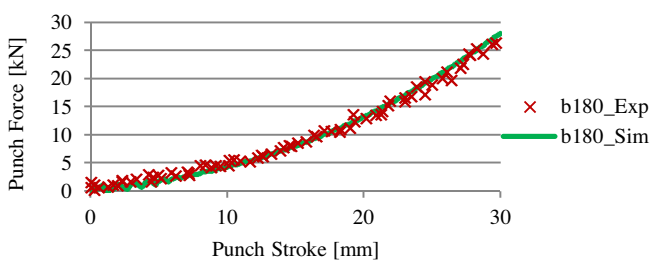

b100

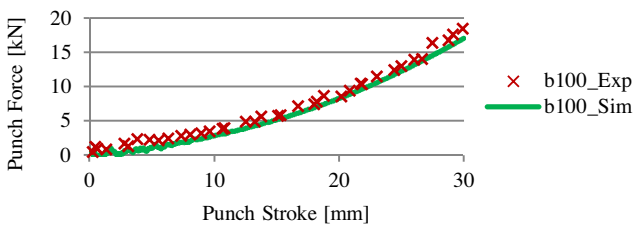

b65

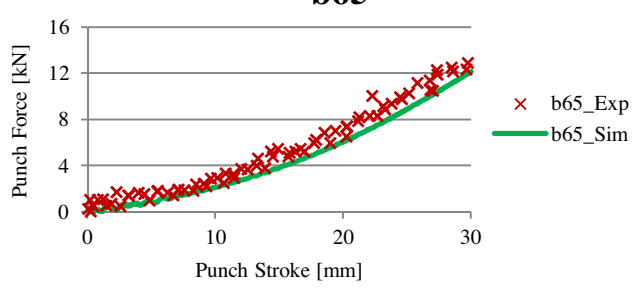

b30

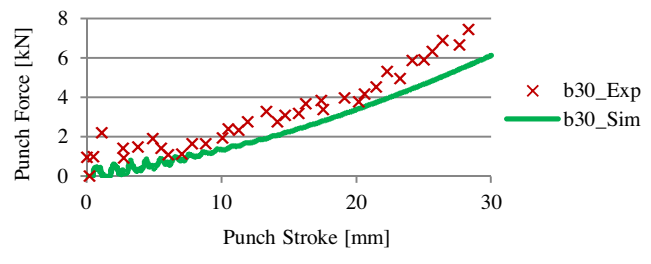

b20

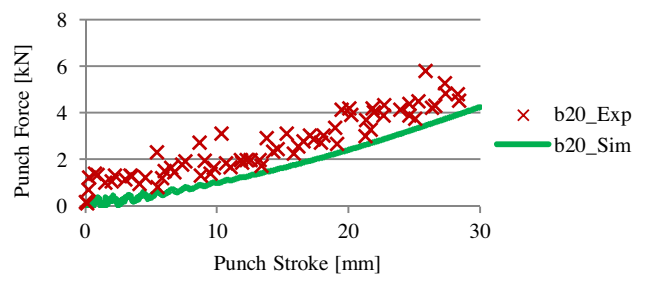

Fig. 12. Comparison of real and numerical punch forces during Nakazima test to each specimen geometry.

With all the constitutive parameters identified and the "unknown" core stress-strain curve deduced, the material model can be built. This new strategy was effective in provide the missing data to model the mechanical behavior of the Hybrix microsandwich as a multilayer material.

\section{Hybrix: numerical simulations and validation}

In the previous sections, the experimental FLC was defined and the constitutive parameters of the yield criterion for each layer were identified. Now, the hardening laws must be identified from the stress-strain data. The hardening curve identified for the core is graphically shown in Fig. 7.

The swift law was chosen to model the isotropic hardening curve of the stainless steel skins, and the parameters identified as graphically shown in Fig. 8. 


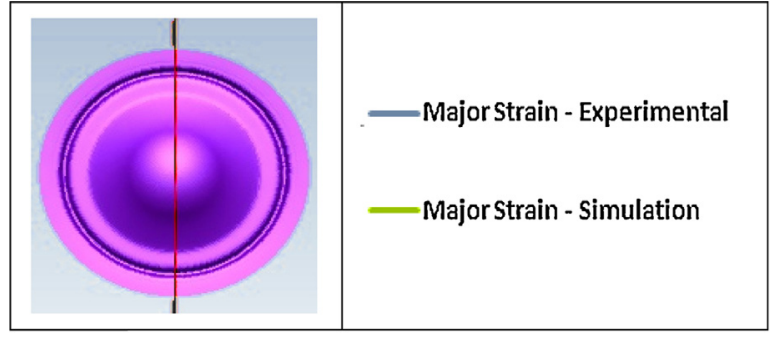

Fig. 13. Cross-section for major strain evaluation of all Nakazima specimens.

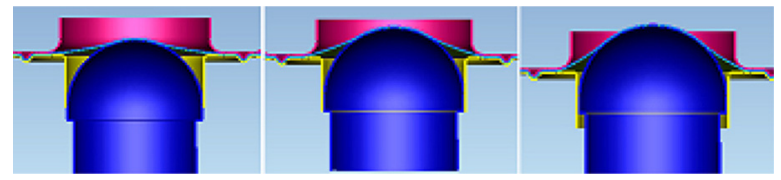

Fig. 14. Different evaluation stages.

Taking into account the hardening curves of the core [plotted in Fig. 7] and of the metallic skins [plotted on Fig. 8] the hardening behavior of the total Hybrix can be simulated. The Swift parameters of the skin were adjusted until the best fit between the Hybrix average force-displacement curve experimentally measured for $0^{\circ}$ [Fig. 2] and the one determined numerically. The comparison between experimental and numerical curves is shown in Fig. 9.

Comparing the standard yield stress values of the AISI 304L steel, i.e. between $260 \mathrm{MPa}$ and $280 \mathrm{MPa}$, with the values experimentally measured from the skin's tensile samples with a nominal thickness of $0.15 \mathrm{~mm}$, i.e. around $135 \mathrm{MPa}$, the main conclusion is that the values experimentally determined are much lower than the expected (or standard) ones. However, such conclusion is in good agreement with uniaxial tensile tests performed on very thin stainless steel samples (nominal thicknesses smaller than $0.3 \mathrm{~mm}$ ), for which the stress-strain curves are sistematically under the ones obtained for samples with larger thicknesses. Besides, the role of even small edge deffects increases when the nominal thicknesses decreases. This so-called "size effect", i.e. the variation of the mechanical properties of the stainless steel when the sheet's nominal thickness decreases was well described and documented by Geiger et al. (1997) in micro-forming, and Chen et al. (2009) in micro-bending. Hoffmann and Hong (2006) also investigated the grain size effect in copper foils whereas Diehl et al. (2008) focused upon aluminium sheets. Since it is proven a degradation of mechanical properties for very thin sheets, it is not recommended to use stress-strain curves of tensile samples with higher thicknesses to characterize the mechanical behavior of the very thin skins.

Concerning the elastic properties, and given that the punch forces determined numerically for the Nakazima test were very low, the value of the Young modulus was adjusted until a good fit between experimental and numerical forces was attained. The main results are shown in Table 4, i.e. the values of the Young modulus determined from the experimental uniaxial tensile tests and the ones determined from the adjustment between numerical and experimental Nakazima tests

The values of the experimental Young modulus is also very far from the theoretical value usually adopted for the steel, i.e. $210 \mathrm{GPa}$. Even considering a degradation of the elastic properties for very small thicknesses, it seems that conventional tensile tests are unreliable to measure the Young modulus in case of micro-sandwich materials. However, the skin to core Young modulus ratio obeys to the rule of mixtures and it is a key-parameter to adjust the elastic properties of the micro-sandwich sheet as stated in Crolla (2015).

For modeling the multi-layer Hybrix material, 3D 8-node hexagonal solid finite element meshes were used [Fig. 10]. The FE mesh comprises 4 layers of elements, 2 layers for the two exterior metallic skins and 2 layers for the composite core.

In the virtual tensile test, the experimental $50 \mathrm{~mm}$ length gauge was mimicked by following the displacement of 2 nodes initially at the same relative distance, as schematically shown in Fig. 11.

The CAD geometry of the Nakazima tools were obtained with a 3D scanner and converted in IGES format to PAM-STAMP.

\section{Discussion and comparison}

In this section experimental and numerical results are analyzed and compared. To validate the accuracy of the numerical model, the results of Nakazima tests, namely the forming forces, the major strain distribution and the formability are compared.

\subsection{Forming forces}

Punch displacement and punch force were recorded during Nakazima tests. The comparison between experimental and simulation results is presented in Fig. 12 for several geometries of the Nakazima specimen.

In case of Nakazima tests, the specimen geometries with higher values of the punch force, namely the b180, b100 and b65 geometries, exhibit smaller oscillations in terms of the experimental punch force evolution. On the contrary, noisy curves can be seen in case of b30 and b20 specimen geometries, as shown in Fig. 12. The reason for this behavior can be related with the load cell sensitivity. Therefore, in order to minimize the contribution of the noisy curves, the adjustment procedure between experimental and numerical curves was focused mainly on the noisyless curves.

\subsection{Major strain distribution}

Major strain field distribution was also analyzed in the outer surface (the outside surface of the metallic skin not in contact with the punch) along the well-defined line identified in Fig. 13. The major strain fields are determined from ARAMIS software. The same line was defined and used in PAM-STAMP $2 \mathrm{G}$ in order to allow the comparison between experimental and numerical results.

Three time steps were selected for comparison purposes, corresponding to a punch displacement of around $10 \mathrm{~mm}, 20 \mathrm{~mm}$ and $30 \mathrm{~mm}$, as identified in Figs. 14 and 15 .

At the first time step (i.e. at about $10 \mathrm{~mm}$ of punch displacement) the experimental major strains are always higher than the numerical major strains. Moreover, this difference is larger for the narrower specimens b30 and b20. Such behavior can be explained by the existence of pre-strain that is not taken into account equally in the experimental and numerical simulations. In fact, the cameras just began recording the Nakazima test after the blankholder closing, and so the reference configuration was not effectively the initially one. In fact, the blankholder closing introduces some prestrains in the sample, what is more pronounced in case of the narrower samples. As the punch displacement increases the experimental and numerical values close up. The better correlation is achieved for the larger punch displacement Fig. 15.

\subsection{Formability}

Sheet metal forming simulation is undoubtedly a valuable support for the development of manufacturable sheet metal parts. During this process, the evaluation of part and process feasibility is strongly dependent of the formability study, which can predicted the risk of ruptures and/or splits, the wrinkling tendency and zones of insufficient stretching. However, this study can only be done with the Forming Limit Diagram if the FLC is available. 


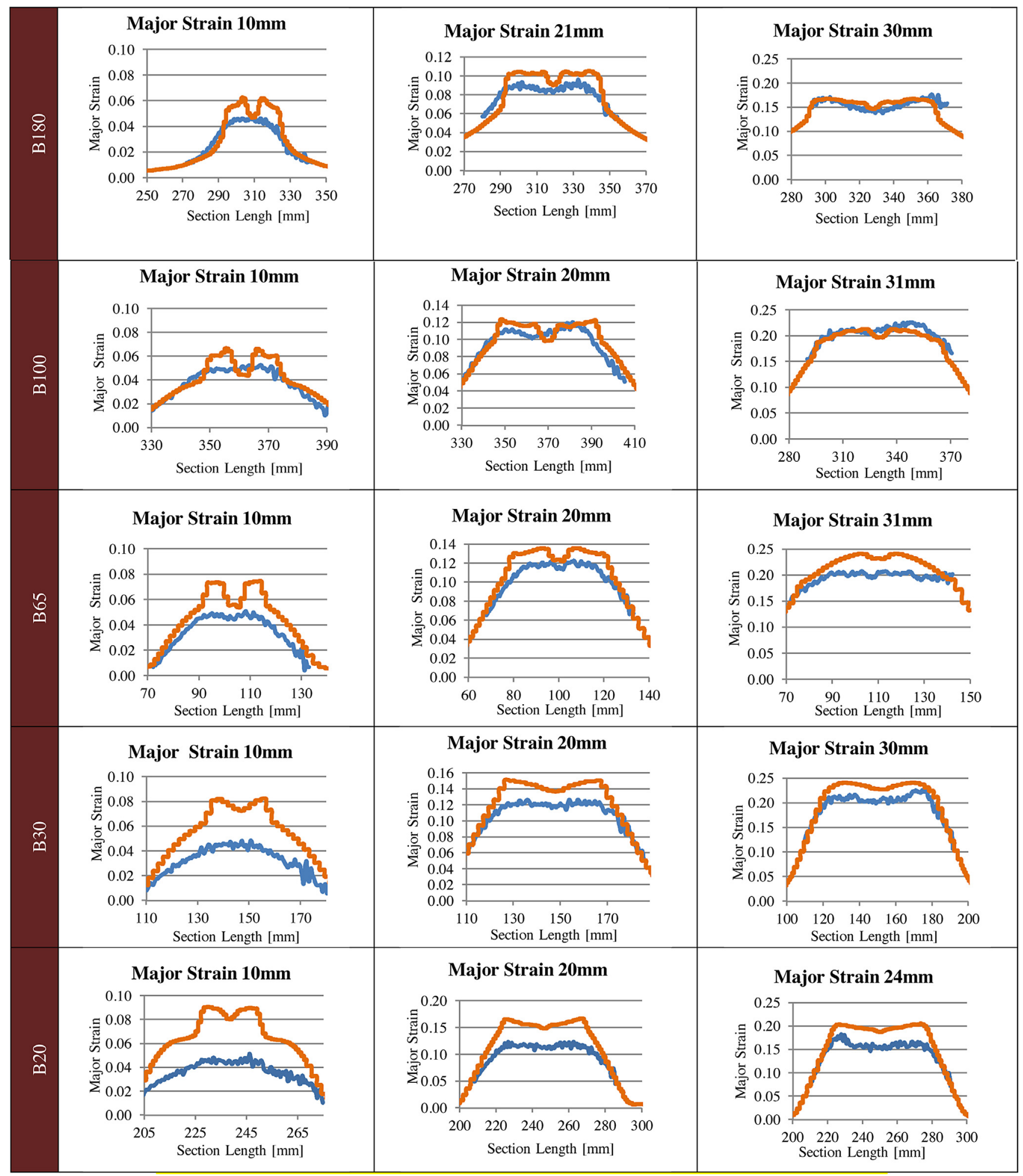

Fig. 15. Comparison between real and numerical major strain distribution, in the same cross-section, to all specimen's geometries.

In general, experimental FLC's are more reliable than mathematical FLC's. As mentioned before, because of the phenomenon of progressive tearing failure in the narrower specimens, the experimental results were unstable. This failure mode is caused by the combination of sheet thickness and the position of the edge of the specimen according to the die radius. As observed by Pepelnjak and Barisic (2009), for sheet thicknesses below $0.4 \mathrm{~mm}$ the undesired tearing take place in the specimens defining the left-hand side of the FLD. Therefore, a mathematical FLC was used for the formability assessment of Hybrix. For each layer, the FLC was automatically generated by the software based on the thickness and the hardening coefficient of the material. The analytical method behind this FLC approximation was developed by Keeler and Brazier (1977).

The average of maximal punch displacements in the experimental Nakazima tests is used as a reference for each geometry, and compared with numerical results for the same conditions. The real 


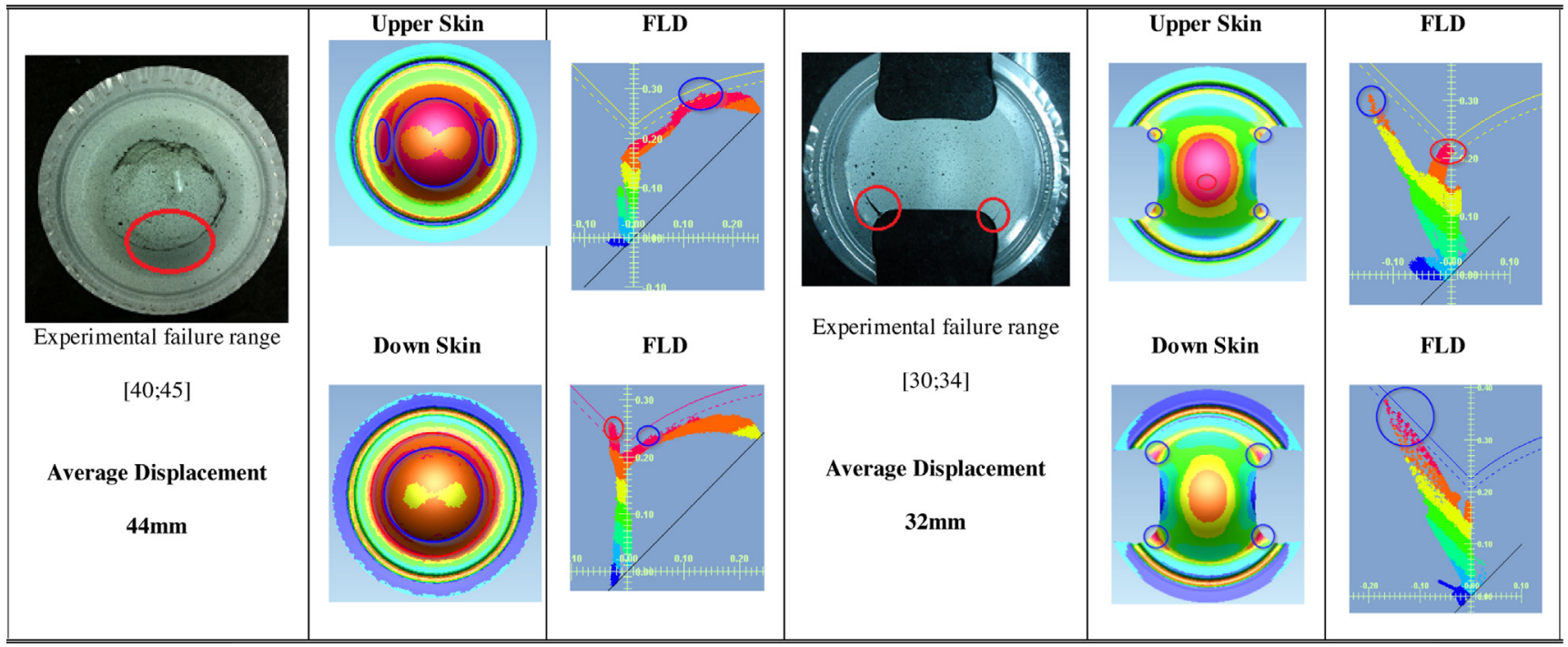

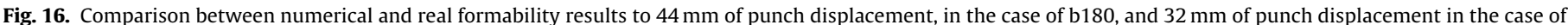
b100.

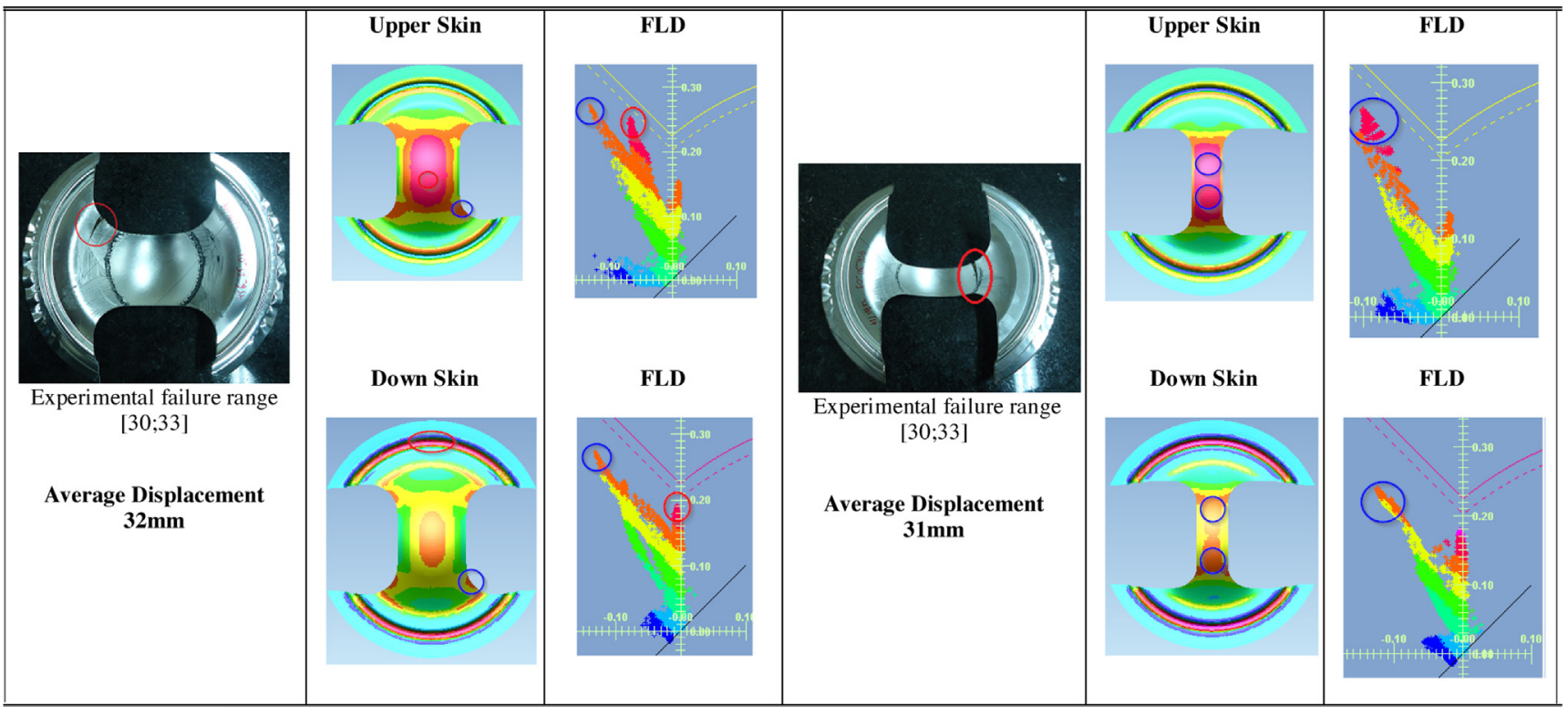

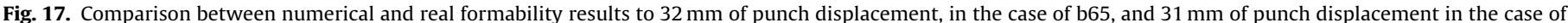
b30.

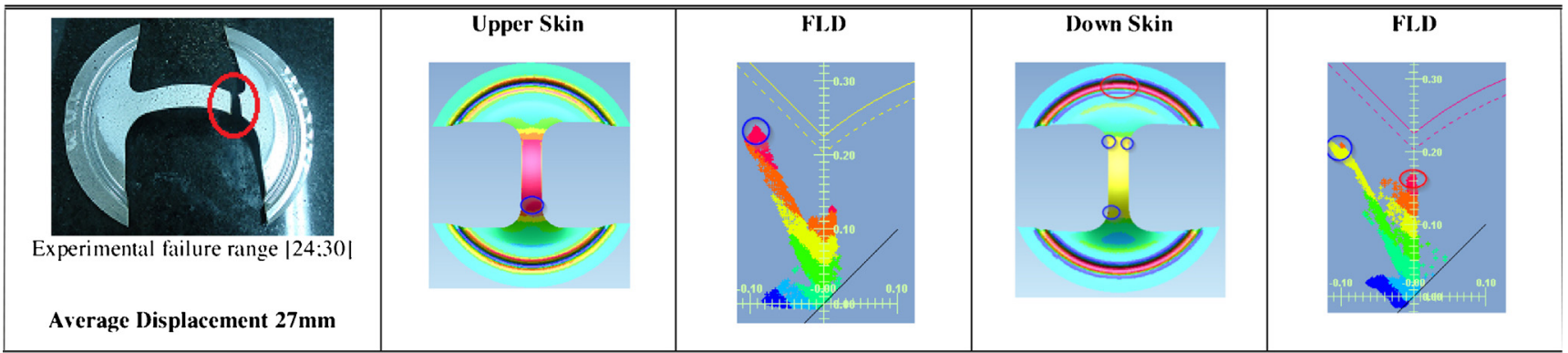

Fig. 18. Comparison between numerical and real formability results in the specimen b20 to $27 \mathrm{~mm}$ of punch displacement. 
critical points were compared with the critical zones in the simulation and marked in blue. Other critical zones in the simulation were marked in red [see Figs. 16-18].

Hot spots can be easily detected in the FLD. The critical zones must be analyzed in both upper and bottom skins in order to obtain an accurate prediction of the specimen failure. In case of specimens b20 and b30, the FLC cannot predict the exact moment (punch displacement) of the specimens' failure. As shown in Fig. 17 and Fig. 18, the cloud of points is still above the FLC, i.e. on the safety region, at the maximum experimental punch displacement. However, in these cases the tearing phenomenon is the mechanism responsible for failure. For this very thin sheets, the geometry of the specimens, the size effects and any micro-defect in the contour (due to the specimens cutting process, for example) can lead to a micro-crack, and early propagation and rupture. So, this Keeler FLC can be still correct and useful. In fact, for many stamping processes, cutting and trimming operations occur only after the stamping operations.

In summary, for general applications, the results shown above shows that this numerical model predicts accurately critical zones and points of rupture.

\section{Hybrix: conclusions}

A simple methodology for experimental characterization of an advanced micro-sandwich material was presented. The method is based on uniaxial tensile tests commonly used for the characterization of metallic sheets. The unknown mechanical behavior of the core was deducted from the overall mechanical behavior of both the Hybrix and of the single metallic skins.

The uniaxial tests performed with total Hybrix specimens did not lead to reliable elastic properties. Nakazima tests allowed to adjust the Hybrix's Young modulus to achieve better results. However, Nakazima tests were not very effective to obtain Hybrix FLC. The fact that metallic skins are too thin, combined with the shape of the specimens, result in tearing failure outside the central area of the specimens, which define the left-hand side of the FLD. In order to eliminate the tearing effect observed in Hybrix the specimens redesign should be considered.

Conclusively, the good correlation between the numerical results and the experimental results in terms of forming forces, major strain distribution and general formability, proves that the proposed methodology is appropriate and functional to develop accurate numerical models for the modeling and simulation of micro-sandwich materials.

\section{Acknowledgments}

The authors greatly acknowledge the financial support of "Fundação para a Ciência e Tecnologia" (FCT-Portugal), through the research project SFRH/BDE/51189/2010 ("Development FEA Tools Applied to Sheet Forming Special Cases. Application to the Automotive Industry, Advanced Metallic Materials and Multi-Layer and Multi-Material Sheets") in partnership with Sodecia Tecnical Center (Maia, Portugal). The authors would also like to thank the company
Lamera AB (Sweden), for providing the Hybrix material, and ESI GROUP to the technical support with PamStamp 2G 2012.2 and collaboration in this study.

\section{References}

Banabic, D., 2010. Sheet Metal Forming Processes: Constitutive Modelling and Numerical Simulation, 1st ed. Springer, pp. 45-52, http://dx.doi.org/10.1007/ 978-3-540-88113-1, ISBN: 978-3-540-88112-4.

Chen, F.K., Shih, W.C., Tu, K.Y., 2009. Micro-bending of thin stainless steel sheets. 6th International Conference on Multi-Material Micro Manufacture, 337-340, http://dx.doi.org/10.3850/4M2009RP001_9070.

Crolla, D., 2015. Materials and manufacturing-sandwich materials. In: Palkowski, H., Sokolova, O.A., Carradó, A. (Eds.), Encyclopedia of Automotive Engineering. John Wiley \& Sons, Ltd., pp. 1-17, http://dx.doi.org/10.1002/9781118354179. auto163, Online 2014.

Diehl, A., Staud, D., Engel, U., 2008. Investigation of the mechanical behaviour of thin metal sheets using the hydraulic bulge test. In: Proceedings of the 4th International Conference Multi-Material Micro Manufacture 4M'2008, Cardiff, pp. 195-198.

Engelmark, M., 2009. Usability Evaluation of the Fibrous Core Sandwich Material Hybrix for Automobile Body Applications. Master of Science Thesis, Stockholm, Sweden, pp. 5-6.

Geiger, M., Mebner, A., Engel, U., 1997. Production of microparts-size effects in bulk metal forming. Similarity Theory Prod. Eng 4 (1), 55-58.

Ghassemieh, Elaheh, 2011. Materials in Automotive Application, State of the Art and Prospects, New Trends and Developments in Automotive Industry. In: Chiaberge, Marcello (Ed.). InTech, http://dx.doi.org/10.5772/13286, ISBN: 978-953-307-999-8.

Hoffmann, H., Hong, S., 2006. Tensile test of very thin sheet metal and determination of flow stress considering the scaling effect. CIRP Annals-Manuf. Technol. 55 (1), 263-266.

Jackson, K.P., Allwood, J.M., Landert, M., 2008. Incremental forming of sandwich panels. J. Mater. Process. Technol. 204, 290-303.

Keeler, S.P., Brazier, W.G., 1977. Relationship between laboratory material characterization and press-shop formability. Microalloying, 75. Union Carbide, pp. 517-530.

Luzin, V., Banovic, S., Gnäupel-Herold, T., Prask, H., Ricker, R.E., 2005. Measurement and calculation of elastic properties in low carbon steel sheet. Mater. Sci. Forum 495-497, 1591-1596.

Mann, D., 1999. Automotive Plastics \& Composites-Worldwide Markets \& Trends to 2007,2 nd ed. Elsevier, pp. 90

Moreira, R.A.S., Sousa, R.J.A., Valente, R.A.F., 2010. A solid shell layerwise finite element for non-linear geometric and material analysis. Compos. Struct. 92 (15), 7-23.

Palkowski, H., Lange, G., 2005. Ame austenitic sandwich materials in the focus of research. Metal.-J. Metall. 11, 215-224.

Pauchard, A., 2009. Precise thin metal cutting using the Laser MicroJet. Laser Elektronikprod. Feinwerkstech. 15 (2009), 145-156.

Pepelnjak, T., Barisic, B., 2009. Computer-assisted engineering determination of the formability limit for thin sheet metals by a modified Marciniak method. J. Strain Anal. 44 (1), 459-472, http://dx.doi.org/10.1243/03093247JSA503.

Starman, B., Vrh, M., Halilovič, M., Štok, B., 2014. Advanced modelling of sheet metal forming considering anisotropy and youngčs modulus evolution. J. Mech. Eng. 60 (2), 84-92, http://dx.doi.org/10.5545/sv-jme.2013.1349.

Van Den Boscha, M.J., Schreursa, P.J.G., Geersa, M.G.D., 2009. On the prediction of delamination during deep-drawing of polymer coated metal sheet. J. Mater. Process. Technol. 209, 297-302, http://dx.doi.org/10.1016/j.jmatprotec.2008. 02.024 .

\section{Web references}

jtmconsultancy, 2015. https://www.jtmconsultancy.nl. TSE, 2014. https://www.thyssenkrupp-steel-europe.com/en/ press/press-releases/press-release-6552.html. 